\title{
Psychological Implications of Cleft Lip and Palate (CLP) in Children
}

\author{
Asif Iqbal Khan, M.D.S., ${ }^{\prime}$ Ashok K. Utreja, M.D.S., ${ }^{2}$ Roobal Behal, M.D.S.' \\ 'Government Dental College and Hospital, Srinagar, India, ${ }^{2}$ Unit of Orthodontics, Post Graduate Institute of Medical Education and \\ Research, Chandigarh, India.
}

\section{A B S T R A C T}

BACKGROUND: Although quality of life (QOL) is becoming an important outcome measure in research on children with CLP in Western countries, there is very little information available on this aspect in children with CLP in India.

OBJECTIVE: To determine the self-concept and QOL of children with cleft lip and palate (CLP) patient and to compare it with age matched non CLP patients.

METHODS: Self-concept and QOL were assessed using the Self Concept Scale (SCS) and Pediatric Quality of Life inventory. Total of 160 subjects were divided into 4 age groups of each containing 40 subjects i.e., group 1 (7-10 years), group 2 (1 1 13 years), group 3 (14-16years) and group 4 (16-24years). In each age group, 20 were CLP subjects and the other 20 were non CLP subjects

RESULTS: The CLP subjects had lower self-concept scores than the non-CLP subjects. The self-concept scores showed a decreased trend in CLP subjects across the group from age 7-10 years to subjects $>16$ years. The quality of life score in CLP subjects was lower than that in the non CLP subjects. A statistically significant lower quality of life score was seen in adult cleft group compared to non CLP group.

CONCLUSION: The self-concept and quality of life scores were significantly lower in older age CLP subjects compared to non CLP subjects. JMS 2012;15(2):115-18

Key words: Cleft lip and palate, psychosocial, self-concept, QOL

Clefts of the lip and palate are by far the most common major congenital malformations of face. The prevalence of CLP is 1 in 500 to 1000 live births world wide, with highest incidence in Afghans (4.9 per 1000 live births) and lowest in Negroid (1 in 3000). ${ }^{1}$ In India from hospital-acquired data, the incidence is 1.97 per 1000 live births. ${ }^{2}$ The psychosocial variables play a pivotal role in functioning of an individual with CLP. Subjects with CLP therefore require an interdisciplinary approach for proper management and rehabilitation. Despite the interdisciplinary approach of treatment, an

\section{Correspondence:}

Dr. Asif lqbal Khan

Bismillah Braces Centre, near Al Ameen Hospital, Hyderpora

Byepass, Airport Road, Srinagar, Kashmir, India-190014

E-mail: drasiforthol@yahoo.co.in approach to get into the insight of psychosocial variables among subjects with CLP is not explored adequately. It is likely that prevalence of psychological problems might be higher than what the literature suggests, since only $20 \%$ of cleft teams worldwide had carried out psychological assessment of either the patients. In subjects with CLP, diverging results from lowered self-concept to a medium to high selfconcepts $^{2-4}$ have been documented. Studies have also shown that persons with facial disfigurement had lower quality of life than the persons without deformity. ${ }^{5}$ Overall aspects such as well-being and social life are affected but not the more practical and tangible aspects of their daily life. ${ }^{6}$ Although health related quality of life is becoming an important outcome measure in research on the children with CLP in the Western countries, there is so far no published report on 
this aspect in children with CLP in India. Thus the present study was conducted to determine self-concept and quality of life of subjects with CLP and to compare it with age matched non CLP subjects.

\section{Methods}

The cross sectional study was carried out in the Unit of Orthodontics, Oral Health Sciences Center in collaboration with Department of Psychiatry and Clinical Psychology, Department of Plastic surgery and Department of Pediatrics, Post Graduate Institute of Medical Education and Research, Chandigarh, India.

The sample consisted of 160 subjects who were divided into 4 age groups i.e., group 1 to 4 in the age range of 7-10 years, 11-13 years, 14-16 years and 16-24 years respectively. Each age group consisted of 40 subjects, out of whom 20 were in experimental group (with CLP) and the other 20 were non CLP subjects served as control group. Demographic variables like age, sex, education, locality, family, cleft repair, orthodontic treatment, orthognathic surgery as well as socioeconomic status were noted. Subjects having only isolated cleft palate, mental retardation, any syndrome and organic diseases were excluded from the study.

The psychological implications of CLP on affected subjects were determined by Self-Concept Scale (SCS)7and Pediatric Quality of Life Inventory.

The self-concept scale used in this study was a local language version of Piers Harris children self-concept scale containing 80 items of having 'yes or no' response. It was used to measure self-concept of subjects having CLP and to compare it with non CLP children. This test was a verbal paper pencil test and had six subscales i.e., behavior, intellectual and school status, physical appearance and attributes, anxiety, popularity and happiness, and satisfaction. Total items of six subscales were recorded on an answer sheet and then total self-concept scale score was obtained by adding all items.

The pediatric quality of life inventory was used to evaluate the quality of life of subjects with CLP and to compare it with non CLP subjects. The pediatric quality of life inventory measurement model measures the health related quality of life and integrate seamlessly both generic score scales and disease specific modules into one measurement system. The pediatric quality of life inventory generic score has four scales containing 23 items and measures the core dimensions of health as delineated by the WHO. These four scales included were physical functioning, emotional functioning, social functioning, and school functioning. Among 23 total items, 8 items were in physical health summary score and rest 15 were in psychosocial health summary score. In addition to these scores, 12 new items were constructed and categorized into 4 main domains i.e. speech, social participation, appearance and eating domains. To complete the questionnaire, each child was asked how much of a problem in each item had been during the past one month and the children themselves after receiving proper instructions filled the questionnaires. A 5-point scale was used to rate the severity of items (0-never, 1-almost never, 2sometimes, 3-often and 4-almost often). On the pediatric quality of life inventory generic core scales, items were scored in a reverse manner and then linearly transformed to a $0-100$ scale and higher score indicated better health related quality of life. For reverse scoring, $0-4$ scale items were transformed to $100-0$ as $0=100,1=75,2=50,3=25$ and $4=0$. Adding the scores of all items then made the total mean quality of life score.

The data was statistically analyzed by SPSS (version 12) software. All data was subjected to descriptive statistics. Differences among the groups for demographic profiles were analyzed using chi-square test.

ANOVA (analysis of variance) was used for comparison of means between groups and within groups. "t-test" was used to compare CLP and non CLP subjects in age groups of 7-10 years,11-13 years,14-16 years and 16-24 years for Self Concept scores and Quality of Life.

\section{Results}

The results of the demographic variables are discussed in table 1 . All the demographic profiles except family type of the CLP and non CLP subjects were comparable. In group 4 subjects, greater number of non CLP subjects belonged to nuclear families than with CLP and that was significant statistically $(p<0.05)$.

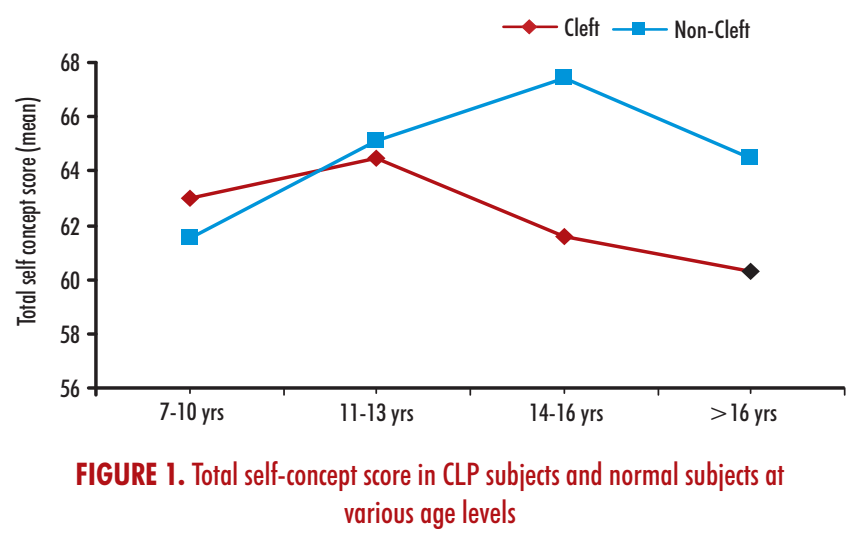

The self-concept scores of different groups are shown in figure 1. In general, the self-concept scores decreased from group 1 to 4 in subjects with CLP where as in non CLP subjects the scores were increased from group 1 to 3 and then reduced in group 4. The self-concept score in group 1 CLP subjects was more than that in the group-1 non CLP subjects and the difference was statistically non-significant $(p \geq 0.05)$. The self-concept score of 14-16 years non CLP subjects was significantly more than that in the subjects with CLP $(p<0.05)$. 
TABLE 1. Various Demographic variables among Cleft Lip Palate (CLP) and non CLP (non CLP) subjects.

\begin{tabular}{|c|c|c|c|c|c|c|c|c|c|c|c|c|c|}
\hline & & \multicolumn{3}{|c|}{ Group 1 (7-10 years) } & \multicolumn{3}{|c|}{ Group 2 (11-13 years) } & \multicolumn{3}{|c|}{ Group 3 (14-16 years) } & \multicolumn{3}{|c|}{ Group 4 (>16years) } \\
\hline & & CLP & Non CLP & p-value & CLP & Non CLP & p-value & CLP & Non CLP & $\mathrm{p}$-value & CLP & Non CLP & $p$-value \\
\hline Age (mean and SD) & & $8.7+0.8$ & $8.4+1.1$ & 0.45 & $12.1+0.86$ & $11.9+0.68$ & 0.38 & $14.9+0.86$ & $15.9+0.99$ & 0.42 & $19.3+1.19$ & $19.4+2.03$ & 0.43 \\
\hline \multirow[t]{2}{*}{ Sex } & Male & 14 & 9 & \multirow[b]{2}{*}{0.10} & 11 & 12 & & 10 & 12 & \multirow[b]{2}{*}{0.16} & 11 & 14 & \multirow[b]{2}{*}{0.16} \\
\hline & Female & 6 & 11 & & 9 & 8 & 0.26 & 10 & 8 & & 9 & & \\
\hline \multirow[t]{5}{*}{ Education of parent } & Graduate & Nil & Nil & \multirow{5}{*}{0.09} & Nil & Nil & & Nil & Nil & & 10 & 16 & \multirow{5}{*}{0.19} \\
\hline & High & Nil & $\mathrm{Nil}$ & & $\mathrm{Nil}$ & Nil & & $\mathrm{Nil}$ & $\mathrm{Nil}$ & & 8 & 4 & \\
\hline & Middle & 15 & 19 & & 16 & 15 & 0.17 & 15 & 1 & 0.57 & $\mathrm{Nil}$ & & \\
\hline & Primary & 19 & 15 & & 1 & 4 & & 5 & 4 & & 1 & Nil & \\
\hline & Uneducated & Nil & Nil & & Nil & Nil & & Nil & 1 & & Nil & Nil & \\
\hline \multirow[t]{3}{*}{ Locality } & Urban & 13 & 14 & \multirow{3}{*}{0.50} & 10 & 14 & & 16 & 14 & \multirow{3}{*}{0.35} & 10 & 15 & \multirow{3}{*}{0.19} \\
\hline & & & & & & & 0.16 & & & & & & \\
\hline & Rural & 7 & 6 & & 10 & 6 & & 4 & 6 & & 10 & 5 & \\
\hline \multirow[t]{3}{*}{ Family } & Nuclear & 17 & 12 & \multirow{3}{*}{0.07} & 13 & 14 & & 15 & 11 & \multirow{3}{*}{0.16} & 101 & 7 & \multirow{3}{*}{$0.02 *$} \\
\hline & & & & & & & 0.50 & & & & & & \\
\hline & Joint & 3 & 8 & & 7 & 6 & & 5 & 9 & & 10 & & \\
\hline \multirow[t]{4}{*}{ Status } & High & 1 & Nil & \multirow{4}{*}{0.48} & Nil & 1 & & Nil & 2 & & Nil & Nil & \multirow{4}{*}{0.31} \\
\hline & Upper middle & e 4 & 6 & & 6 & 7 & 0.54 & 8 & 3 & 0.10 & 8 & 5 & \\
\hline & Lower middle & e 15 & 14 & & 14 & 12 & & 12 & & 15 & 11 & 15 & \\
\hline & Lower & Nil & Nil & & Nil & Nil & & Nil & & Nil & 1 & Nil & \\
\hline
\end{tabular}

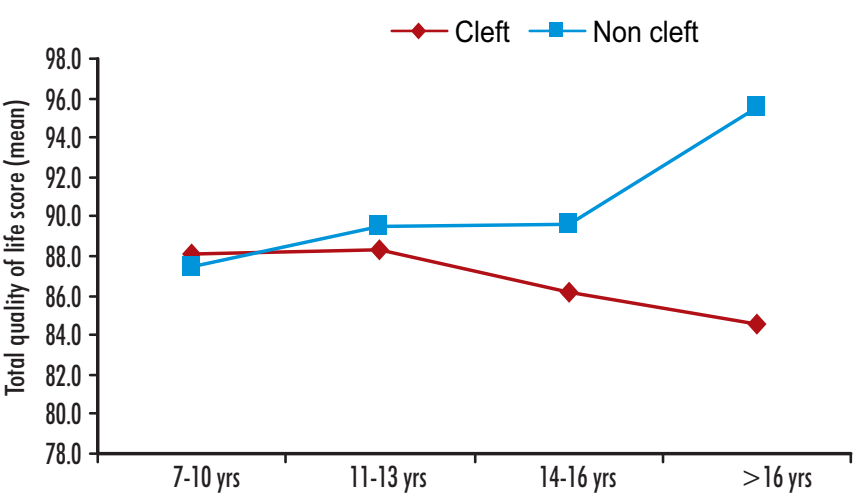

FIGURE 2. Total quality of life score in CLP subjects and normal subjects at various age

The total quality of life score in cleft and non CLP subjects at various age levels is shown in figure 2 . The quality of life score in subjects with CLP was less than in non CLP subjects. In both CLP and non CLP subjects, the quality of life score showed decreased trend from subjects of group 1 to 4 , and after 16 years of age a statistically highly significant $(p<0.001)$ difference in the score was found.

\section{Discussion}

The results of the present study showed no significant differences in the self-concept scores in most age groups of the subjects with CLP as compared to that of the non CLP subject groups. However, in the late adolescent cleft subjects with CLP (age 14-16 years) the self-concept score was significantly lower. This was in contrast to the study of Brantley and Clifford(1979), ${ }^{9}$ where he found adolescent group with cleft had high self-concept when compared to non CLP adolescents. However, Kapp ${ }^{10}$ reported no difference in the self-concept among cleft and non CLP subjects. Thus the present study revealed that patients with CLP could do as well similar to the non CLP population. There was no consistent pattern in the attitude of cleft subjects, which might be due to varied demographic variables or some other reason that may need further investigation. The mean total self-concept score in the cleft patients decreased across the groups as we moved up from primary school age children to adolescence and young adults. This indicted that age was a significant factor by itself in determining the direction of selfconcept, contrary to the earlier studies in which age alone was not important. ${ }^{3}$ The maturation of the person, exposure to the outside world as well as the concept of attractiveness might contribute significantly in lowering the self-concept scores.

Appearance was often mentioned as being problematic for the subjects with the CLP. However, in the present study, this factor was isolated from personal attribute. All groups had lower scores for appearance than attributes indicating the importance of good looks in overall personality development. Also, in a society where physical attractiveness is desired and thought to promote personality and improve social skills, it becomes natural that it increases the concern and affects the self-concept. ${ }^{10}$ 
The quality of life is an especially important health outcome to assess in children with the CLP because they are high-risk group and in critical development period during which cognitive and social skills have to be learnt. In the present times, the effectiveness of health interventions is being measured by influence on the quality of life of patients. Overall the quality of life in the subjects with CLP was lower than in non CLP subjects for almost all age groups, which reached to a significant difference in adult age group (age $>16$ years). The results indicated that as the age of the patients with CLP increased they were more dissatisfied and the quality of life had suffered showing a decreased trend across the cleft groups. The cleft group indicated more marked and selective effects than did the group without cleft. Overall aspects such as well-being and social life were affected but not the more practical and tangible aspect of daily living, for example, bathing, running and doing household chores. This was in accordance to the study of Marcusson, Akerlind and Paulin $^{6}$ who also reported no major effects on quality of life of these patients. One important finding in the present study was that the perceived physical health score was higher in subjects with CLP than non CLP in the age group of 7-10 years to 14-16 years, which could have resulted in better psychosocial functioning and so a better quality of life in early age groups.

In the present study male subjects with CLP had better overall quality of life than the girls. This could be due to the fact that the girls might have greater concerns of aesthetic problems or it could be that the boys may hide their scars or get involved in other competent activities which might have influenced the quality of life score.

Longitudinal studies involving subjects with the clefts and age and gender matched controls are needed to identify the duration of psychosocial problems and to determine whether specific developmental stages were influential in the occurrence of these problems. It is important to determine thoroughly whether problems experienced by the children with CLP continue into adulthood or perhaps only emerge in adulthood. Studies that could take into account the influence of facial growth, which is not complete until the late teenage years, and children and adolescents with CLP may undergo a number of surgical procedures during this time. This will provide more insight of psychosocial issues in these patients. Facial growth changes and the experience of surgery should also be examined as possible factors influencing psychosocial function. If we are to identify those most at risk from psychosocial problems and to determine the extent of such problems, then these factors require further investigation.

In conclusion, the number of non CLP subjects belonging to nuclear family was significantly more than the number of CLP subjects in group 4 (age 16-24 years). The self concept was less in the subjects of CLP than that of non CLP subjects. The self-concept of subjects with CLP decreased as the age increased. The quality of life was better in non CLP subjects than that of subjects with CLP. In CLP subjects, the quality of life became worse as the age increased where as in non CLP subjects the quality of life improved as the age increased.

\section{References}

1. Turner SR, Rumsey N, Sandy JR. Psychological aspects of CLP. EurJ Orthod 1998;20:407-415.

2. Kapp-Simon K. Self concept of primary school age children with cleft clip, cleft palate, or both. Cleft PalateJ 1986;23:24-27.

3. Goffman E. Encounters: Two studies in the sociology of interaction, Bobbs- Merrill Co. Inc., Indianapolis. 1963.

4. Persson M, Aniasson G, Becker M, Svensson H. Self concept and introversion in adolescents with CLP. Scand J Plas Reconst Surg Hand Surg 2002;36:24-27.

5. Tisza , Veronica B, Gumpertz E. The parent's reaction to birth and early care of children with cleft palate. Pediatr 1962;30:86-90.

6. Marcusson A, Akerlind I, Paulin G. Quality of life in adults with repaired CLP. Cleft Palate Craniofac J 2001; 38:379-381.

7. Ahluwalia SP. A manual for children self concept scale. National Psychological Corporation.1971.

8. Bref A, Field D. WHOQOL-BREF. Introduction, administration, scoring and generic version of assessment, Field trial version.1996.

9. Brantley HT, Clifford E. Cognitive, self - concept and body-image measures of non CLP, cleft palate, and obese adolescents. Cleft Palate J 1979;16:177-82.

10. Kapp K. Self-concept of the cleft lip and or palate child. Cleft Palate J 1979;16:171-76. 